\title{
A Notion of Harmonic Clustering in Simplicial Complexes
}

\author{
Stefania Ebli ${ }^{1}$ and Gard Spreemann ${ }^{1}$ \\ ${ }^{1}$ Laboratory for Topology and Neuroscience, École Polytechnique Fédérale de Lausanne, 1015 Lausanne, Switzerland
}

October 17, 2019

\begin{abstract}
We outline a novel clustering scheme for simplicial complexes that produces clusters of simplices in a way that is sensitive to the homology of the complex. The method is inspired by, and can be seen as a higher-dimensional version of, graph spectral clustering. The algorithm involves only sparse eigenproblems, and is therefore computationally efficient. We believe that it has broad application as a way to extract features from simplicial complexes that often arise in topological data analysis.
\end{abstract}

\section{Introduction}

An important objective in modern machine learning, and part of many scientific and data analysis pipelines, is clustering 4 . By clustering, we generally mean the separation of data into groups, in a way that is somehow meaningful for the domain-specific relationships that govern the underlying data and problem in question. However, the demands that the clustering scheme should satisfy are of course inherently vague.

For data that form a point cloud in Euclidean space, and where one expects $k$ clusters to exist, one may employ elementary methods such as $k$-means clustering 37 . For data in a more abstract "similarity space", for which no obviously meaningful Euclidean embedding exists, researchers invented the schemes 35,34 that we today refer to as hierarchical clustering. Alternatively, one can derive a graph structure from some notion of similarity between the data points. Treating the data points as vertices of a graph allows one to exploit the popular and highly successful spectral clustering techniques [41, 30] which developed from the field of spectral graph theory 9 .

Although the graph structure provides us with additional information about the data, graphs are intrinsically limited to modeling pairwise interactions. The success of topological methods in studying data, and the parallel establishment of topological data analysis (TDA) as a field [13, 42] (see also [6, 7, 12, 15 for modern introductions and surveys), have confirmed the usefulness of viewing data through a higher-dimensional analog of graphs [28, 32]. Such a higher-dimensional analog is called a simplicial complex, a mathematical object whose structure can describe $n$-fold interactions between points. Their ability to capture hidden patterns in the data has led to various applications from biology 16 ,
33] to material science 21. Recent work has also expanded classical graph-centric results — such as Cheeger inequalities [17, 5, isoperimetric inequalities [31] and spectral methods 22 - to simplicial complexes. This leads naturally towards a novel domain of "spectral TDA" methods.

In this paper we present the harmonic clustering algorithm, a novel clustering scheme inspired by the wellknown spectral clustering algorithm for graphs. Our method, like spectral clustering, does not require any parameter optimization and involves only computing the smallest eigenvalue eigenvectors of a sparse matrix. The harmonic clustering algorithm is applied directly to a simplicial complex and it outputs a clustering of the simplices (of a fixed degree) that is sensitive to the homological structure of the complex, something that is highly relevant in TDA. Moreover, since simplices can encode interactions of higher order than just the pairs captured by graphs, our algorithm allows us to cluster complex community structures rather than just the entities they comprise.

Our method can be seen as complimentary to the one presented in [5].

\subsection{Spectral graph theory}

The method we present in this paper does not require many formal results from spectral graph theory. The notions relevant for our purposes are described below for the sake of completeness.

In its simplest form, the Laplacian of an undirected and unweighted finite graph $G$ is taken to be the positive definite matrix $L=D-A$, where $A$ is the adjacency matrix of $G$ and $D$ its diagonal degree matrix (i.e. the row/column sums of $A$ ). The normalized Laplacian is then defined as $\bar{L}=D^{-1 / 2} L D^{-1 / 2}$. For reasons that will become clear later on (see 2.1), we will write $C_{0}(G)$ for the free real vector space generated by the vertices of $G$, and consider $L$ as the matrix of a linear map $C_{0}(G) \rightarrow C_{0}(G)$ in this basis.

Already in the middle of the 19th century it was clear that the eigenvalue spectrum of $L$ has a lot to say about $G$, as is evident from as early as a historic theorem of Kirchhoff relating the eigenvalues of the Laplacian with the number of spanning trees in the graph 25. From the 1950s, graph theorists and quantum chemists were independently discovering more relationships between a graph and the eigenspectrum of its Laplacian. However, 
the publication of the book 10 may be said to mark the start of spectral graph theory as a field in its own right. A modern introduction to the field and references to the results listed below can be found in [9].

The spectrum of $L$ encodes information about the connectivity of the graph. For instance, the number of connected components of the graph is equal to the dimension of the kernel of $L$. Moreover, the eigenvectors associated to the zero eigenvalues, also called harmonic representatives, take constant values on connected components. A perhaps more interesting result is given by the Cheeger constant [8], a measure of how far away a connected graph is from being disconnected by bounding the smallest non-zero eigenvalue of $L$.

Theorem 1 (Cheeger, 1969 [8]; see also e.g. [9]). Let $G=(V ; E)$ be a finite, connected, undirected, unweighted graph. Write cut $(G)$ for the triples $(S, \bar{S}, \partial S)$ with $S, \bar{S} \subseteq$ $V$ and $\partial S \subseteq E$ such that $S \sqcup \bar{S}=V$ and

$$
\partial S=\{(u, w) \in E: u \in S, w \in \bar{S}\} .
$$

Define the Cheeger constant of $G$ as

$$
h(G)=\min _{(S, \bar{S}, \partial S) \in \operatorname{cut}(G)} \frac{|\partial S|}{\min \left(\sum_{u \in S} \operatorname{deg}(u), \sum_{w \in \bar{S}} \operatorname{deg}(w)\right)} .
$$

Then the first non-zero eigenvalue $\lambda_{1}$ of the graph's normalized Laplacian satisfies

$$
2 h(G) \geq \lambda_{1} \geq \frac{(h(G))^{2}}{2} .
$$

A partition of $V$ as $S \sqcup \bar{S}$ that attains the Cheeger constant is called a Cheeger cut. It is known that finding an exact Cheeger cut is an NP-hard problem [38]. One of the best known approaches to approximating the Cheeger cut is the spectral clustering method, which takes the first non-zero eigenvalue eigenvector of the graph Laplacian as a relaxed real-valued solution of the original discrete optimization problem 41. Namely, the smallest nonzero eigenvector of $\bar{L}$, also called the Fiedler vector or the connectivity vector [14], can be exploited to find the best partition of the graph into two "almost disconnected" components. The Cheeger cut can be easily generalized to find $k+1$ "almost disconnected" components using the $k$ first non-zero eigenvectors of the graph Laplacian [41.

\section{$1.2 \quad$ Graph spectral clustering}

The Fiedler vector being a relaxed solution of the Cheeger cut has implications for clustering the vertices of a graph into "almost disconnected" components 41, 3]. For the remainder of this section we will assume that the graph under consideration is connected.

Graph spectral clustering of a graph $G=(V, E)$ with Laplacian $L$ works in two steps. First, one uses the information encoded in the lowest-eigenvalue eigenvectors of $L$ to map $V$ into low-dimensional Euclidean space. One thereafter uses standard $k$-means or any applicable Euclidean clustering technique on the points in the image of this map, before pulling back to $G$. Specifically, we will write $e_{1}, e_{2}, \ldots, e_{n}$ for the eigenvectors associated with the $n$ first non-zero eigenvalues of $L$. One defines a function, also called a spectral embedding, $\phi: C_{0}(G) \rightarrow$ $\mathbb{R}^{n}$ by

$$
\phi(v)=\left(\left\langle v, e_{1}\right\rangle,\left\langle v, e_{2}\right\rangle, \ldots,\left\langle v, e_{n}\right\rangle\right)
$$

where $\langle\bullet, \bullet\rangle$ is the inner product on $C_{0}(G)$ that makes $V$ orthonormal. As a finite Euclidean point cloud, $\operatorname{im} \phi$ is then clustered in $\mathbb{R}^{n}$ by standard $k$-means or any suitable clustering algorithms. The clustering obtained is then pulled back to $V$. Figure 1 shows an example. Observe that in this case, mapping into the real line using only the Fiedler vector would suffice (i.e. $n=1$ ).

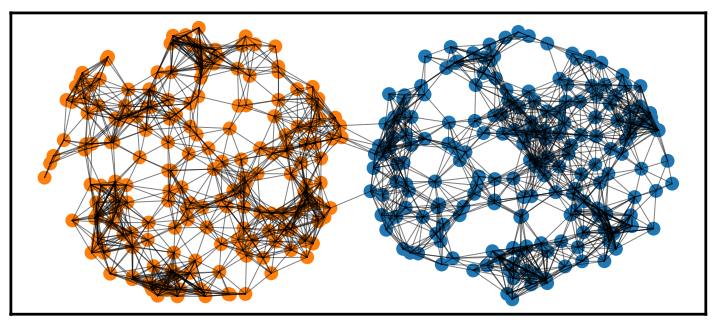

Figure 1: Graph spectral clustering of the nodes of a graph with two well-connected components weakly interconnected. Clustering using the Fiedler vector produces as clusters the well-connected components.

As pointed out in 41], spectral clustering is one of the standard approaches to identify groups of "similar behavior" in empirical data. It is therefore not surprising that it has been successfully employed in many fields ranging from computer science and statistics to biology and social science. Moreover, compared to other approaches, such as Gaussian Mixture Models clustering, spectral clustering does not require any parameter optimization and can be solved efficiently by standard linear algebra methods.

\section{Harmonic clustering in simpli- cial complexes}

Our method is inspired by spectral clustering in graphs, but applies instead to a higher-dimensional analog, namely simplicial complexes. Instead of clustering only vertices, which are the zero-dimensional building blocks of graphs and simplicial complexes, the method clusters independently building blocks of any dimension.

This section outlines the prerequisite basic constructions from algebraic topology before describing our method. A reader interested in more background on algebraic topology is directed to standard textbooks 18 .

Those wishing a quick overview of method can view it in algorithmic form in figure 3

\subsection{Algebraic topology}

A simplicial complex is a collection of finite sets closed under taking subsets. We refer to a set in a simplicial complex as a simplex of dimension $p$ if it has cardinality $p+1$. Such a $p$-simplex has $p+1$ faces of dimension $p-1$, namely the sets omitting one element, which we will denote as $\left(v_{0}, \ldots, \hat{v}_{i}, \ldots, v_{p}\right)$ when omitting the $i$ 'th 
element. While this definition is entirely combinatorial, we will soon see that there is a geometric interpretation, and it will make sense to refer to and think of 0 -simplices as vertices, 1-simplices as edges, 2-simplices as triangles, 3 -simplices as tetrahedra, and so forth.

Let $C_{p}(K)$ be the free real vector space with basis $K_{p}$, the set of $p$-simplices in a simplicial complex $K$. The elements of $C_{p}(K)$ are called $p$-chains. These vector spaces come equipped with boundary maps, namely linear maps defined by

$$
\begin{aligned}
& \partial_{p}: C_{p} \rightarrow C_{p-1} \\
& \partial_{p}\left(\left(v_{0}, \ldots, v_{p}\right)\right)=\sum_{i=0}^{p}(-1)^{i}\left(v_{0}, \ldots, \hat{v}_{i}, \ldots, v_{p}\right)
\end{aligned}
$$

with the convention that $C_{-1}(K)=0$ and $\partial_{0}=0$ for convenience. Figure 2 shows how the boundary maps give a geometric interpretation of simplicial complexes.

One readily verifies that $\partial_{p} \circ \partial_{p+1}=0$, and so $C \bullet(K)$ is a real chain complex. By the $p$ 'th homology vector space of $K$ we will mean the $p$ 'th homology vector space of this chain complex, namely

$$
H_{p}(K)=H_{p}\left(C_{\bullet}(K)\right)=\operatorname{ker} \partial_{p} / \operatorname{im} \partial_{p+1} .
$$

The elements of ker $\partial_{p}$ are called $p$-cycles, while those of im $\partial_{p+1}$ are called $p$-boundaries, as can be seen geometrically in Figure 2. The Betti numbers are the dimensions of the homology vector spaces, and we write $\beta_{p}(K)=\operatorname{dim} H_{p}(K)$. Intuitively, the Betti numbers count connected components, non-bounding loops, nonbounding cavities, and so forth.

We emphasize again that this is homology with real coefficients, not integer or finite field, as is common in TDA.

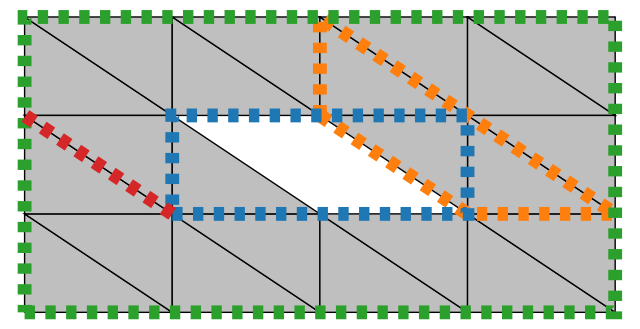

Figure 2: A simplicial complex $K$ with 20 0-simplices, 38 1-simplices (the edges) and 22 2-simplices (the filled triangles), with some highlighted 1-chains. The highlighted simplices in these represent the edges with non-zero coefficient in each chain (the unfamiliar reader is invited to fill in possible values for these coefficients). The red 1-chain consists of a single 1-simplex, and is neither a cycle nor a boundary. The orange 1-chain has trivial boundary, and is therefore a cycle. It is not a representative of any non-trivial homology class, for it is the boundary of 2-chain consisting of the three 2-simplices it encloses. The green and the blue 1-chains are cycles that represent the same homology class (intuitively the 2-dimensional hole in the middle). $H_{0}(K)$ is 1-dimensional, $K$ 's single connected component, while $H_{1}(K)$ is 1-dimensional due to the central hole.

\subsection{Simplicial Laplacians}

We are in this paper concerned with finite simplicial complexes, and assume that they are built in a way that encodes useful information about the data being studied.
We will briefly discuss the case where each simplex in $K$ comes equipped with extra data - including, but not limited to the filtration/weighting information that is ubiquitous in TDA — or with a normalization factor derived from the complex's structure, in the form of a function $w: K \rightarrow \mathbb{R}_{+}$. The latter is analogous to the various normalization schemes that are often used in graph spectral theory. Our computational experiments, however, will only consider the case $w=1$.

The weights are encoded into the chain complex by endowing each degree with the inner product that makes all simplices orthogonal, and a simplex have norm given by the weight, i.e.

$$
\begin{aligned}
& \langle\bullet, \bullet\rangle_{i}: C_{i}(K) \times C_{i}(K) \rightarrow \mathbb{R} \\
& \langle\sigma, \tau\rangle_{i}= \begin{cases}w(\sigma)^{2} & \text { if } \sigma=\tau \\
0 & \text { otherwise. }\end{cases}
\end{aligned}
$$

Further discussions of weighting schemes can be found in 22 .

We place no further assumptions on the simplicial complex that we take as input. In particular, it is not necessary for it to come equipped with some embedding into Euclidean space, nor do we demand that it triangulates a Riemannian manifold. Therefore dualities like the Hodge star, which is used to construct the Hodge-de Rham Laplacian in the smooth setting [26] that motivates us, are unavailable for our method. The same is true for discrete versions of the Hodge star, such as that of Hirani 20. Instead of dualizing with respect to a Hodge star, to define a discrete version of the Laplacian for simplicial complexes, we simply take the linear adjoint of the boundary operator with respect to the inner product, defining $\partial_{i}^{*}: C_{i-1} \rightarrow C_{i}$ by

$$
\left\langle\partial_{i}^{*} \sigma, \tau\right\rangle_{i}=\left\langle\sigma, \partial_{i} \tau\right\rangle_{i-1} \quad \forall \sigma \in K_{i-1}, \tau \in K_{i} .
$$

In analogy with Hodge-de Rham theory, we then define the degree- $i$ simplicial Laplacian of a simplicial complex $K$ as the linear operator $\mathcal{L}_{i}: C_{i}(K) \rightarrow C_{i}(K)$ such that

$$
\begin{aligned}
& \mathcal{L}_{i}=\mathcal{L}_{i}^{\text {up }}+\mathcal{L}_{i}^{\text {down }} \\
& \mathcal{L}_{i}^{\text {up }}=\partial_{i+1} \circ \partial_{i+1}^{*}: C_{i}(K) \rightarrow C_{i}(K) \\
& \mathcal{L}_{i}^{\text {down }}=\partial_{i}^{*} \circ \partial_{i}: C_{i}(K) \rightarrow C_{i}(K) .
\end{aligned}
$$

The harmonics are defined as

$$
\mathcal{H}_{i}(K)=\operatorname{ker} \mathcal{L}_{i} .
$$

Observe that there are $p$ Laplacians for a complex of dimension $p$. In most practical applications, the matrices for the Laplacians are very sparse and can easily be computed as a product of sparse boundary matrices and their transposes.

The following discrete version of the important Hodge decomposition theorem is a simple exercise in linear algebra in the current setting.

Theorem 2 (Eckmann, 1944 [11]). The vector spaces of chains decompose orthogonally as

$$
C_{i}(K) \cong \mathcal{H}_{i}(K) \oplus \operatorname{im} \partial_{i+1} \oplus\left(\operatorname{ker} \partial_{i}\right)^{\perp} .
$$

Moreover, 
1. $\mathcal{H}_{i}(K) \cong H_{i}(K)$

2. the harmonics are both cycles and cocycles (i.e. cycles with respect to $\partial_{i+1}^{*}$ )

3. the harmonics are the $L^{2}$-minimal representatives of their (co)homology classes, i.e. if $h \in \mathcal{H}_{i}(K)$ and $h \sim z \in \operatorname{ker} \partial_{i}$ are homologous, then $\langle h, h\rangle_{i} \leq\langle z, z\rangle_{i}$.

The first detailed work on the spectral properties of this kind of simplicial Laplacian was carried out by Horak and Jost [22]. Recently Steenbergen et al. [36] provided a notion of a higher dimensional Cheeger constant for simplicial complexes. At the same time, Gundert and Szedlák [17] proved a lower bound for a modified version of the higher dimensional Cheeger constant for simplicial complex which was later generalized to weighted complexes by Braxton et al. Mukherjee and Steenbergen 29 developed an appropriate notion of random walks on simplicial complexes, and related the asymptotic properties of these walks to the simplicial Laplacians and harmonics. It is worth mentioning that, to the best of our knowledge, no connection between the eigenvectors of the simplicial Laplacian and an optimal cut for simplices in higher dimensions is known.

Our contribution is a notion of spectral clustering for simplicial complexes using the harmonics.

\subsection{Harmonic clustering}

Observe that the ordinary graph Laplacian, as described in section 1.1. is just the matrix of $\mathcal{L}_{0}=\mathcal{L}_{0}^{\text {up }}$ in the standard basis for $C_{0}(G)$. The function $\phi$ in equation (1) can thus be seen as projecting the 0 -simplices onto a subspace of low-but-nonzero-eigenvalue eigenvectors of $\mathcal{L}_{0}$. The zero part of the spectrum is not used. Theorem 2 makes the reason clear: harmonics in $\mathcal{H}_{0}(G)$ have the same coefficient for every vertex in a connected component of $G$. As connectivity information is easy to obtain anyway, there is little use in adding these eigenvectors to the subspace that $\phi$ projects onto. This is not so for the higher Laplacians. In fact, our method primarily uses the harmonics, and only optionally ventures into the non-zero part of the eigenspectrum.

In what follows, $K$ is a fixed simplicial complex arising from data. The particulars of how $K$ was built from data is outside the scope of this paper, and is a topic that is well-studied in the field of TDA in general. Our goal is to obtain a useful clustering of $K_{p}$ for some chosen $p$. We assume that $K$ is of low "homological complexity" in degree $p$, by which we mean that $\beta_{p}(K)$ is small (less than 10, say).

Analogously to $\phi$ above, we define the harmonic embedding

$$
\begin{aligned}
& \psi: K_{p} \rightarrow \mathbb{R}^{\beta_{p}(K)} \\
& \psi=\xi \circ \operatorname{proj}_{\mathcal{H}_{p}(K)} \circ i,
\end{aligned}
$$

where $i: K_{p} \hookrightarrow C_{p}(K)$, proj : $C_{p}(K) \rightarrow \mathcal{H}_{p}(K)$ is orthogonal projection, and $\xi: \mathcal{H}_{p}(K) \rightarrow \mathbb{R}^{\beta_{p}(K)}$ is any vector space isomorphism. In practice, we simply pick an orthonormal basis $h_{1}, \ldots, h_{\beta_{p}(K)}$ for $\mathcal{H}_{p}(K)$ and let

$$
\psi(\sigma)=\left(\left\langle\sigma, h_{1}\right\rangle_{p},\left\langle\sigma, h_{2}\right\rangle_{p}, \ldots,\left\langle\sigma, h_{\beta_{p}(K)}\right\rangle_{p}\right) .
$$

In many situations of practical use, it turns out that many points in $\operatorname{im} \psi$ lie along one-dimensional subspaces of $\mathbb{R}^{\beta_{p}(K)}$. The membership of a point $\psi(\sigma)$ in such a subspace is what is used to cluster the $p$-simplex $\sigma$ (or to leave it unclustered in case it is not judged to be sufficiently close to lying in one of the subspaces). This amounts to clustering $K_{p}$ by performing Euclidean subspace clustering of im $\psi$. A variety of Euclidean subspace clustering methods are available, but are outside the scope of this paper. Examples include independent component analysis [23], SUBCLU 24], and density maximization on $\mathbb{S}^{\beta_{p}(K)-1}$ (or, more precisely, on $\mathbb{R P}^{\beta_{p}(K)-1}$ ), which itself has a multitude of approaches, including purely TDAbased ones by means of persistent homology of sublevel sets.

We point out that the choice of the isomorphism $\xi$ : $\mathcal{H}_{p}(K) \rightarrow \mathbb{R}^{\beta_{p}(K)}$ does not matter on a theoretical level. It may, however, have practical implications for how easy it is to perform subspace clustering. In experiments we typically choose $\xi$ to be the isomorphism that sends $h_{i}$ to the standard basis vector $e_{i}$. Choosing a different orthonormal basis for $\mathcal{H}_{p}(K)$ then just amounts to an element of $\mathrm{SO}\left(\beta_{p}(K)\right)$ acting on $\operatorname{im} \psi$.

Figure 3 summarizes our method in algorithmic form.

Require: Integer $p \geq 0$; simplicial complex $K$ with $\beta_{p}=\operatorname{dim}\left(H_{p}(K)\right)$ small, $K_{p}=\left\{\sigma_{1}, \ldots, \sigma_{N}\right\}$, and inner products $\langle\bullet, \bullet\rangle_{p}$ on $C_{p}(K)$.

$L_{p} \leftarrow$ matrix for $\mathcal{L}_{p}$

$\left(h_{1}, \ldots, h_{\beta_{p}}\right) \leftarrow$ orthonormal basis for $\operatorname{ker} \mathcal{L}_{p}$ (computed using iterative methods [19] on $L_{p}$ )

$$
\begin{aligned}
& \text { for } i=1 \text { to } i=N \text { do } \\
& \quad x_{i} \leftarrow\left(\left\langle\sigma_{i}, h_{1}\right\rangle_{p}, \ldots,\left\langle\sigma_{i}, h_{\beta_{p}}\right\rangle_{p}\right) \\
& \text { end for } \\
& \left(a_{1}, \ldots, a_{k}\right) \leftarrow \operatorname{subcluster}\left(x_{1}, \ldots, x_{N}\right) \\
& \text { for } i=1 \text { to } i=k \text { do } \\
& \quad c_{i} \leftarrow\left\{\sigma_{j} \in K_{p}: j \in a_{i}\right\} \\
& \text { end for }
\end{aligned}
$$

Ensure: Homologically sensitive clustering $c_{1}, \ldots, c_{k}$ of $p$-simplices in $K$.

Figure 3: Our method in algorithmic form. The subroutine subcluster refers to any Euclidean subspace clustering scheme, such as independent component analysis [23], SUBCLU 24], or density maximization on $\mathbb{S}^{\beta_{p}(K)-1}$ (or, more precisely, on $\mathbb{R P}^{\beta_{p}(K)-1}$ ). The latter can be done using methods from TDA, for example by means of persistent homology of certain sublevel sets. Note that there may be unclustered simplices, i.e. it may happen that $\cup_{i=1}^{k} c_{i} \neq K_{p}$.

\section{Experimental results}

In this section we present experimental results for the harmonic clustering algorithm on synthetic data. Specifically, we focus on clustering the edges of various constructed simplicial complexes. The outcomes of our experiments suggest that the harmonic clustering algorithm provides 
clusters sensitive to the homology of the complex. Comparing our results with those of the traditional spectral clustering algorithm applied to the graph underlying the simplicial complex reinforces the idea that our algorithm reveals substantially different patterns in data compared to the classical method.

Below, we consider four simplicial complexes. Three of them are complexes built from Euclidean point clouds by standard methods from TDA, while one is a triangulation of a torus. We reiterate that our method works with abstract simplicial complexes without utilizing any embedding of these into an ambient space. Euclidean point clouds just happen to be a good and common source of simplicial complexes in TDA, and allow for visualization of the obtained clustering in a way that easily relates to the original data.

An important step in preprocessing many kinds of input data in TDA is constructing a simplicial complex satisfying certain theoretical properties. In particular, if the input data come from points sampled from a topological space $X \subset \mathbb{R}^{n}$, one may wish for the homology of the complex to coincide with the homology of $X$.

Two constructions for which some such guarantees exist are the alpha complex [1] and the Vietoris-Rips (VR) complex 40. Both can be seen as taking a point cloud and producing a filtered simplicial complex $K$, i.e. a sequence $\left(K_{t}\right)_{t \in \mathbb{R}_{+}}$with the property that $K_{s} \subset K_{t}$ whenever $s \leq t$. We wish to work with a single simplicial complex, not a filtration, so we use persistent homology (see e.g. 15]) to find the filtration scale $t$ for which $K_{t}$ has the appropriate homology. Of course, since in practice one probably has little or no knowledge of $X$ itself, one cannot necessarily know the "correct" $t$ to consider. However, it is often the case in TDA that long-lived homological features that is to say, homology classes that remain non-trivial under the induced maps $H_{p}\left(K_{s}\right) \rightarrow H_{p}\left(K_{t}\right)$ for large $t-s$ - express interesting properties of the underlying space. We therefore choose a $K_{t}$ to consider by looking for a scale $t$ within the range of a manageable number of long-lived features and few short-lived ones in the degree under consideration.

In the following experiments, we simplify the setup in the algorithm in figure 3 by performing the subroutine subcluster in a somewhat ad hoc semi-manual way. Specifically, all the images of the $\psi$ 's lie in $\mathbb{R}^{2}$ or $\mathbb{R}^{3}$ in these experiments, so we manually pick out the subspaces $V_{1}, \ldots, V_{k}$ in question. Then, the points in $\operatorname{im} \psi$ are orthogonally projected onto each of the subspaces. A point $\psi(\sigma)$ is determined to lie on subspace $V_{i}$ if $\operatorname{proj}_{V_{i}}(\psi(\sigma) /\|\psi(\sigma)\|)$ has norm at least 0.98 , while the onto all other subspaces has norm less than 0.02. The simplex $\sigma$ is then said to be in cluster number $i$. If the above is not true for any of the subspaces, $\sigma$ is considered unclustered.

In many of the experiments that follow, many points in $\operatorname{im} \psi$ end up determined as "unclustered" because they project well onto neither of the 1-dimensional subspaces, or project too well onto multiple of them, as described in 2.3. This is not necessarily a problem, as the parts that are clustered contain a lot of useful information. Moreover, the problem can be reduced by choosing less ad hoc subspace clustering methods than we are currently employing.

To ease visualization, we focus on simplicial complexes that naturally live in $\mathbb{R}^{2}$ or $\mathbb{R}^{3}$ because they arise from point clouds.

\subsection{Wedge of a sphere and two circles}

In this experiment, we consider a noisy sampling of $X=\mathbb{S}^{2} \vee \mathbb{S}^{1} \vee \mathbb{S}^{1}$ realized as a central unit sphere with unit circles wedged onto antipodal points. We sampled 1000 points uniformly randomly from the central sphere, adding radial uniform noise with amplitude 0.01 . The circles were sampled using 100 points each, again with a radial noise of 0.01 . This yields a point cloud with 1200 points, which is shown in figure 4. The VR complex is certainly a suboptimal choice of simplicial complex to build on this kind of data, but we chose it to demonstrate that our method works well also for such an overly dense complex. The complex, constructed at scale $1 / 2$ and denoted $K$ within this section, has 357221 -simplices and 4851892 -simplices, and the Betti numbers are $\beta_{0}(K)=1$, $\beta_{1}(K)=2, \beta_{2}(K)=1$, as for $X$ itself.

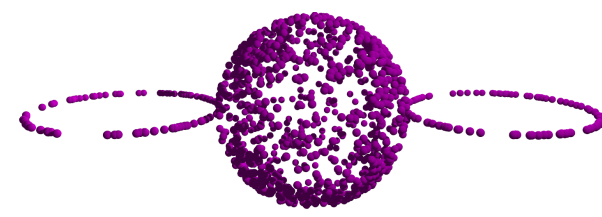

Figure 4: The point sample under consideration in 3.1

We focus on clustering the 1-simplices of the complex. The image of $\psi$ in $\mathbb{R}^{2}$ is shown in figure 5 . The points are colored according to which of the two one-dimensional subspaces they are deemed to belong to. The determination was made by a simple criterion of projecting well enough onto one of the lines, but not the other. Points that project well onto both or neither are considered unclustered and shown as red. Figure 6 shows this clustering pulled back to the complex itself, excluding the unclustered edges. Observe how the method separates the 1-simplices of the VR complex in a manner that is sensitive to the two non-bounding cycles that generate homology in degree 1 (the two circles).

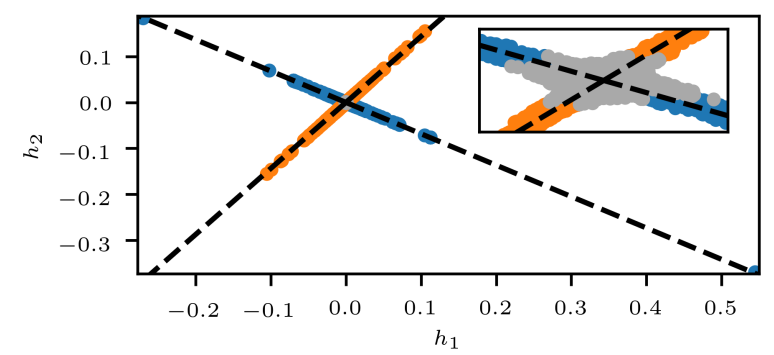

Figure 5: The image of $\psi$ for the 1-simplices in the VR complex from the experiment in 3.1 The dashed lines indicate the subspaces used for clustering. The inset shows a detailed view near the origin, where one can see a large number of points in gray that are unclustered due to them projecting too well onto both subspaces.

We also repeated the experiment with one of the circles in $X$ moved to be attached to the other circle instead of 


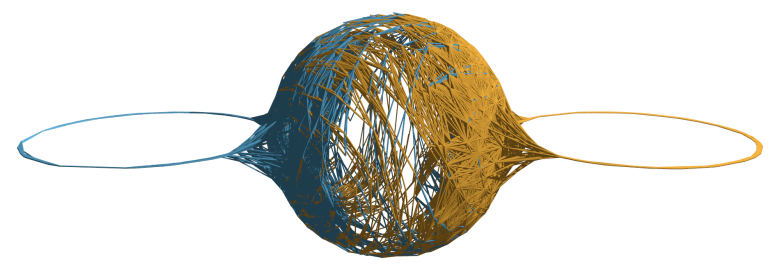

Figure 6: The clustering from figure 5 pulled back to the 1-simplices of the VR complex from 3.1. which is here drawn in $\mathbb{R}^{3}$ using the coordinates of the points for visualization purposes only. The unclustered 1-simplices, 19254 in number, are not drawn.

the sphere. This space is obviously homotopy equivalent to $X$, but is geometrically very different. figure 7 shows the result. Observe that the sphere is now captured by its adjacent circle, and that the unclustered edges tend to be those near where the two circles intersect.

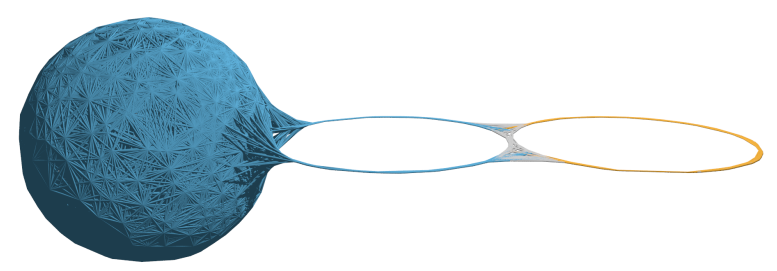

Figure 7: The result of clustering the rearranged point cloud from 3.1 Again the 1-simplices of the VR complex are clustered in a way respecting the generators of 1-homology. The unclustered simplices, 467 in number, are drawn in gray. (That the sphere appears solid is only a visualization artifact; the 2 -simplices are not drawn.)

\subsection{Punctured plane}

In this experiment we uniformly randomly sample 1000 points from a unit square in $\mathbb{R}^{2}$ with three disks of radius $1 / 10$ cut out. The points are seen as faint does in figure 8 We construct the alpha complex at parameter 0.1 , and denote it by $K$ in this section. It has Betti numbers $\beta_{0}(K)=1, \beta_{1}(K)=3$ and $\beta_{i>1}(K)=0$. There are 2914 1-simplices and 1912 2-simplices. We again focus on the 1-simplices for clustering. The codomain of $\psi$ is now $\mathbb{R}^{3}$, and the image is clustered according to three 1-dimensional linear subspaces. The result is shown in figure 8, and we again observe how the obtained clustering occurs with respect to the punctures of the square.

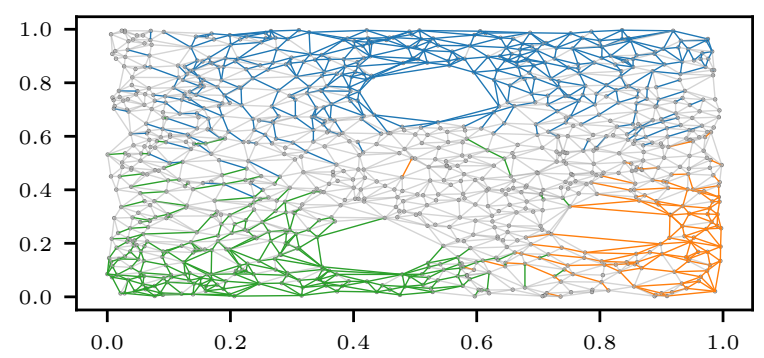

Figure 8: The point cloud of the experiment in 3.2 is shown as faint dots. The punctures can be seen in near $(0.5,0.8),(0.4,0.2)$ and $(0.8,0.3)$, and one observes that the clustered 1-simplices (blue, green, orange, respectively) follow the punctures. The gray 1simplices are unclustered. The 2 -simplices have not been drawn.

\subsection{Torus}

We next perform clustering of the edges of two different tori.

\subsubsection{From a point cloud}

We uniformly randomly sampled 1500 points from the unit square and map these under $(\varphi, \theta) \mapsto$ $((2+\sin (2 \pi \varphi)) \cos (2 \pi \theta),(2+\sin (2 \pi \varphi)) \sin (2 \pi \theta), \cos (2 \pi \varphi))$ to produce a point sample of a torus in $\mathbb{R}^{3}$. The points were then given a uniformly random noise of amplitude 0.01 in both radii. Again a VR complex $K$ was built, at scale 0.8 . It has 352701 -simplices and 3778732 -simplices, and has the homology of a torus, i.e. $\beta_{0}(K)=1$, $\beta_{1}(K)=2, \beta_{2}(K)=1$. VR was chosen in order for the clustering task to be more complicated than in a more orderly alpha complex.

Figure 9 shows the image in $\mathbb{R}^{2}$ of $K_{1}$ under $\psi$. The subspaces for clustering are somewhat harder to make out than before, but they can still be found. The result of clustering by them can be seen in figure 10. Observe that the two clusters respect the two independent unfilled loops of the torus.

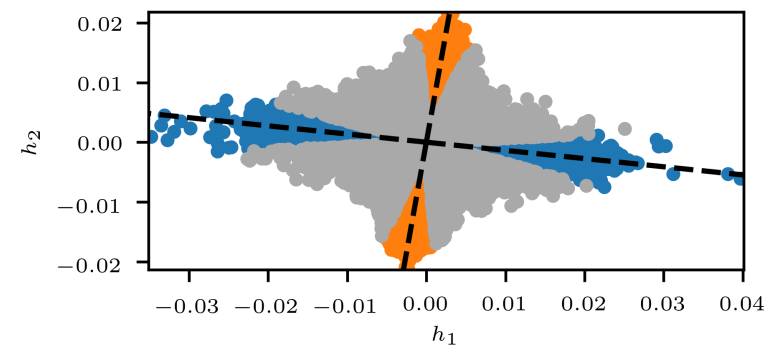

Figure 9: The clustering of the 1-simplices from the simplicial complex obtained from the sampled torus in the experiment in 3.3.1 The unclustered points are shown in gray. They are 23103 in number.

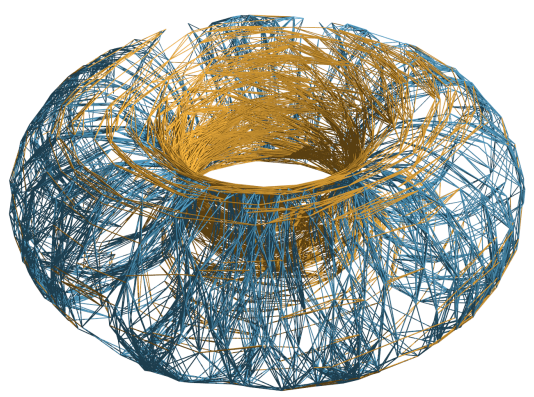

Figure 10: The clustering in figure 9 pulled back to 1-simplices of the torus from the experiment in 3.3.1. The unclustered ones are not shown, something which may make the torus appear broken.

\subsubsection{A triangulation of the flat torus}

As a smaller, more abstract and noise-free example, we consider a triangulation of a flat torus. The considered triangulation consists of a simplicial complex with 9 vertices, 271 -simplices and 182 -simplices. The image of its 1 -simplices in $\mathbb{R}^{2}$ under $\psi$ is shown in figure 11 . The arrangement into a perfect hexagon means that there are 
in fact three subspaces that can be chosen for clustering. The clusters are shown in figure 12 . The arrangement into a hexagon, and therefore the result of three instead of the expected two clusters, disappear if one breaks some of the symmetry in the triangulation, for example by having some of the diagonal edges go the opposite direction.

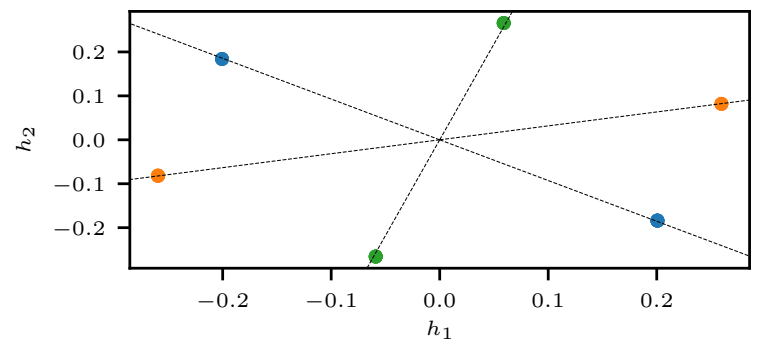

Figure 11: The clustering of the 1-simplices from the simplicial complex obtained as a triangulation of the flat torus from the experiment in 3.3 .2 Note that many points overlap. Three clusters are given by points lying on three different linear subspaces.

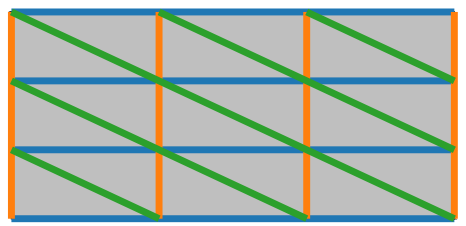

Figure 12: A triangulation of the flat torus represented as a rectangle with pairs of opposing edges identified. The 1-simpilces are clustered into three groups (orange, blue and green). Those in orange and blue are representatives of the two 1-homology classes of the complex, whereas the green ones are a linear combination of the others.

\subsection{Clustering 2-simplices}

We have illustrated our method only on 1-simplices so far for ease of visualization. To point out that it also performs well in other dimensions, we sampled 1000 points (each) from two spheres of radius 1 centered at $(-1,0,0)$ and $(1,0,0)$, each with a radial uniform random noise with amplitude 0.01 . We computed the alpha complex $K$ at parameter 0.3 , so as to create a rather messy region between the spheres. There are 88511 -simplices and 10478 2-simplices, and $\beta_{0}(K)=1, \beta_{1}(K)=0$ and $\beta_{2}(K)=2$ as expected. Our clustering method performs as expected, producing clusters of $K_{2}$ that correspond to homological features, as is shown in figure 13 .

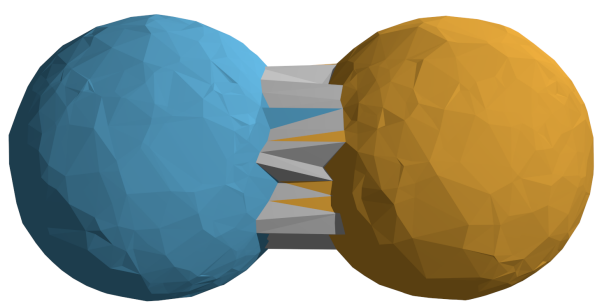

Figure 13: The output of our method when clustering the 2simplices from the complex in the experiment in 3.4 are the blue and orange clusters. The 1485 gray simplices are unclustered.

\subsection{Comparison with graph spectral clus- tering}

It is worth comparing clustering obtained from our method with the ones obtained by clustering the nodes of the graph underlying each simplicial complex using the graph spectral clustering algorithm. Figure 14 shows the results of graph spectral clustering on the nodes of the graph underlying the complex in figure 7. The two first graph Laplacian eigenvectors were used to map the nodes into $\mathbb{R}^{2}$, and then $k$-means was used to find two clusters. Similarly, figure 15 displays three clusters on the nodes of the graph underlying the complex representing a punctured plane with three holes in figure 8. The nodes are mapped to $\mathbb{R}^{3}$ using the three first graph Laplacian eigenvectors, after which $k$-means was used to find three clusters. In both cases we see that the clusters do not reflect any obviously meaningful property of the underlying data, unlike our method, which clusters in a way sensitive to homology.

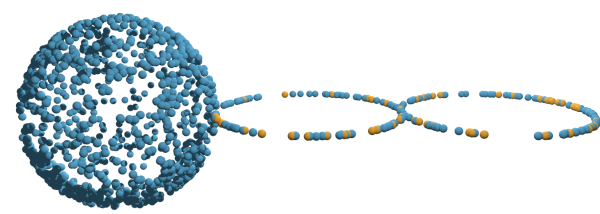

Figure 14: Graph spectral clustering of the vertices of the graph underlying the VR complex of figure 7

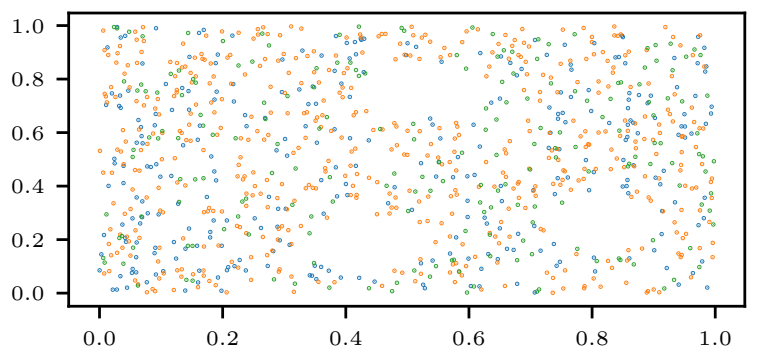

Figure 15: Graph spectral clustering of the vertices of the graph underlying the alpha complex from figure 8

\section{Conclusions and future work}

In this paper we have presented a novel clustering method for simplicial complexes, one that is sensitive to the homology of the complex. We see the method as a contribution to an emerging field of spectral TDA 27, 2]. Our results suggest that the algorithm can be used to extract homological features for simplices of any degree. Experiments in various simplicial complexes demonstrate the ability of the method to accurately detect edges belonging to different non-bounding cycles. Similar results, not shown in this article for practical considerations of visualization, have been obtained by clustering simplices in higher dimensions. The sub-problem of the structure of the linear subspaces in the image of $\psi$, and how to accurately cluster based on demand, require further investigation of both a mathematical and a algorithmic nature.

While our method seems robust to noise in the underlying data, a more thorough investigation into the output's 
dependence on noise, and the output's dependence on the scale at which a point-cloud-derived simplicial complex is built, is warranted.

Moreover, it has not eluded us that the method as outlined is not restricted to clustering just simplices. Other finitely generated chain complexes, such as discrete Morse complexes or cubical complexes, naturally lend themselves to the same analysis. One may also want to consider if there are theoretical implications even in the smooth case.

Further development will also include enlarging the target of the projection in $\psi$ to include non-zero eigenvectors of $\mathcal{L}_{p}$, as in graph spectral clustering. Preliminary results indicate that this yields a further refinement of the homologically sensitive clusters into "fair" subdivisions.

Finally, further work needs to explore the effects of weighting. Both structural weighting, i.e. deriving weights from the local connectivity properties of the complex, as is often done with graph spectral clustering, and weighting originating from the underlying data itself, as is common in TDA.

A potential future application that we suspect fits our method well is collaboration networks $[32]$, where $n$-fold collaborations clearly cannot accurately be encoded as $\left(\begin{array}{l}n \\ 2\end{array}\right)$ pairwise ones.

\section{Acknowledgments}

Alpha complexes and persistent homology were computed using GUDHI 39]. Eigenvector computations were done with $S L E P c$ [19].

We would like to thank K. Hess for valuable discussions.

Both authors were supported by the Swiss National Science Foundation grant number 200021_172636.

\section{References}

[1] Nataraj Akkiraju, Herbert Edelsbrunner, Michael Facello, Ping Fu, EP Mücke, and Carlos Varela. "Alpha shapes: definition and software". In: Proceedings of the 1st International Computational Geometry Software Workshop. Vol. 63. 1995, p. 66.

[2] Sergio Barbarossa, Stefania Sardellitti, and Elena Ceci. "Learning from signals defined over simplicial complexes". In: 2018 IEEE Data Science Workshop (DSW). IEEE. 2018, pp. 51-55.

[3] Mikhail Belkin and Partha Niyogi. "Laplacian eigenmaps and spectral techniques for embedding and clustering". In: Advances in neural information processing systems. 2002, pp. 585-591.

[4] Pavel Berkhin. "A Survey of Clustering Data Mining Techniques". In: Grouping Multidimensional Data. Springer, Berlin, Heidelberg (2006).

[5] Osting Braxton, Palande Sourabh, and Wang Bei. Towards Spectral Sparsification of Simplicial Complexes Based on Generalized Effective Resistance. 2017. arXiv: 1708.08436

[6] Gunnar Carlsson. "Topology and Data". In: Bull. Amer. Math. Soc. 46.2 (2009), pp. 255-308.
[7] Frederic Chazal and Bertrand Michel. An introduction to Topological Data Analysis: fundamental and practical aspects for data scientists. 2017. arXiv: 1710.04019

[8] Jeff Cheeger. "A lower bound for the smallest eigenvalue of the Laplacian". In: Proceedings of the Princeton conference in honor of Professor S. Bochner. 1969, pp. 195199.

[9] Fan R. K. Chung. Spectral graph theory. Vol. 92. Regional Conference Series in Mathematics. American Mathematical Society, 1997.

[10] Dragoš M. Cvetković, Michael Doob, and Horst Sachs. Spectra of Graphs. Theory and Application. Pure and Applied Mathematics. Academic Press, 1979.

[11] Beno Eckmann. "Harmonische Funktionen und Randwertaufgaben in einem Komplex". In: Commentarii Mathematici Helvetici 17.1 (1944), pp. 240-255.

[12] Herbert Edelsbrunner and John Harer. Computational topology: an introduction. American Mathematical Soc., 2010.

[13] Herbert Edelsbrunner, David Letscher, and Afra Zomorodian. "Topological persistence and simplification". In: Proceedings 41st Annual Symposium on Foundations of Computer Science. IEEE. 2000, pp. 454-463.

[14] Miroslav Fiedler. "Algebraic connectivity of graphs". In: Czechoslovak mathematical journal 23.2 (1973), pp. 298305.

[15] Robert Ghrist. "Barcodes: the persistent topology of data". In: Bulletin of the American Mathematical Society 45.1 (2008), pp. 61-75.

[16] Chad Giusti, Eva Pastalkova, Carina Curto, and Vladimir Itskov. "Clique topology reveals intrinsic geometric structure in neural correlations". In: Proceedings of the National Academy of Sciences 112.44 (2015), pp. $13455-13460$.

[17] Anna Gundert and May Szedlák. "Higher dimensional Cheeger inequalities". In: Proceedings of the thirtieth annual symposium on Computational geometry. 2014, p. 181.

[18] Allen Hatcher. Algebraic Topology. Cambridge University Press, 2002.

[19] Vicente Hernandez, Jose E. Roman, and Vicente Vidal. "SLEPc: A scalable and flexible toolkit for the solution of eigenvalue problems". In: ACM Trans. Math. Software 31.3 (2005), pp. 351-362.

[20] Anil N. Hirani. "Discrete Exterior Calculus". PhD thesis. California Insitute of Technology, 2003.

[21] Yasuaki Hiraoka, Takenobu Nakamura, Akihiko Hirata, Emerson G. Escolar, Kaname Matsue, and Yasumasa Nishiura. "Hierarchical structures of amorphous solids characterized by persistent homology". In: Proceedings of the National Academy of Sciences 113.26 (2016), pp. 7035-7040.

[22] Danijela Horak and Jürgen Jost. "Spectra of combinatorial Laplace operators on simplicial complexes". In: Advances in Mathematics 244 (2013), pp. 303-336.

[23] Aapo Hyvärinen and Erkki Oja. "Independent component analysis: algorithms and applications". In: Neural networks 13.4-5 (2000), pp. 411-430. 
[24] Karin Kailing, Hans-Peter Kriegel, and Peer Kröger. "Density-connected subspace clustering for highdimensional data". In: Proceedings of the 2004 SIAM international conference on data mining. SIAM. 2004, pp. 246-256.

[25] Gustav Kirchhoff. "Ueber die Auflösung der Gleichungen, auf welche man bei der Untersuchung der linearen Vertheilung galvanischer Ströme geführt wird". In: $A n$ nalen der Physik 148.12 (1847), pp. 497-508.

[26] Ib Madsen and Jørgen Tornehave. From calculus to cohomology: de Rham cohomology and characteristic classes. Cambridge University Press, 1997.

[27] Joshua L Mike and Jose A Perea. Geometric Data Analysis Across Scales via Laplacian Eigenvector Cascading. 2018. arXiv: 1812.02139.

[28] Terrence J. Moore, Robert J. Drost, Prithwish Basu, Ram Ramanathan, and Anantharam Swami. "Analyzing collaboration networks using simplicial complexes: A case study". In: Proceedings IEEE INFOCOM Workshops (2012), pp. 238-243.

[29] Sayan Mukherjee and John Steenbergen. "Random walks on simplicial complexes and harmonics". In: Random structures $\&$ algorithms 49.2 (2016), pp. 379-405.

[30] Andrew Y. Ng, Michael I. Jordan, and Yair Weiss. "On Spectral Clustering: Analysis and an algorithm". In: Advances In Neural Information Processing Systems. MIT Press, 2001, pp. 849-856.

[31] Ori Parzanchevski, Ron Rosenthal, and Ran J. Tessler. "Isoperimetric inequalities in simplicial complexes". In: Combinatorica 36.2 (2016), pp. 195-227.

[32] Alice Patania, Giovanni Petri, and Francesco Vaccarino. "The shape of collaborations". In: EPJ Data Science 6.1 (2017).

[33] Michael W. Reimann et al. "Cliques of Neurons Bound into Cavities Provide a Missing Link between Structure and Function". In: Frontiers in Computational Neuroscience 11 (2017), p. 48.

[34] Peter H A Sneath. "The application of computers to taxonomy". In: Journal of General Microbiology 17 (1957), pp. 201-226.

[35] Thorvald Sørensen. "A method of establishing groups of equal amplitude in plant sociology based on similarity of species and its application to analyses of the vegetation on Danish commons". In: Biologiske Skrifter 5.4 (1948), pp. 1-34.

[36] John Steenbergen, Caroline Klivans, and Sayan Mukherjee. "A Cheeger-type inequality on simplicial complexes". In: Advances in Applied Mathematics 56 (2014), pp. 5677.

[37] Hugo Steinhaus. "Sur la division des corp materiels en parties". In: Bull. Acad. Polon. Sci 3.4 (1957), pp. 801804.

[38] Arthus Szlam and Xavier Bresson. "Total variation and Cheeger cuts". In: Proceedings of the 27th International Conference on Machine Learning. 2010, pp. 1039-1046.

[39] The GUDHI Project. GUDHI User and Reference Manual. GUDHI Editorial Board, 2015. URL: http://gudhi gforge.inria.fr/doc/latest/.

[40] Leopold Vietoris. "Über den höheren Zusammenhang kompakter Räume und eine Klasse von zusammenhangstreuen Abbildungen". In: Mathematische Annalen 97.1 (1927), pp. $454-472$.
[41] Ulrike von Luxburg. "A tutorial on spectral clustering". In: Statistics and computing 17.4 (2007), pp. 395-416.

[42] Afra Zomorodian and Gunnar Carlsson. "Computing persistent homology". In: Discrete \& Computational Geometry 33.2 (2005), pp. 249-274. 